\title{
Rugged Free-Energy Landscapes - An Introduction
}

\author{
Wolfhard Janke
}

Institut für Theoretische Physik and Centre for Theoretical Sciences (NTZ), Universität Leipzig, Postfach 100 920, D-04009 Leipzig, Germany

wolfhard.janke@itp.uni-leipzig.de

Understanding the static and even more the dynamical behavior of complex physical systems is one of the most challenging problems of current research in physics, chemistry and biology. Paradigmatic examples of condensed matter physics are spin glasses and structural glasses. More recently, in life sciences, the rapid advances in computational biology have added the exploration of the properties of biologically relevant macromolecules to this list. Here, the most prominent example is protein folding, but also studies of protein aggregation and adsorption to soft or hard interfaces are of great medical and technological interest. While the physics concepts underlying the behavior of spin and structural glasses are still quite close, the basic principles governing the properties of biomolecules are in many respects quite diverse. Still, there are certain joint features in all these systems which make a common treatment quite promising for future scientific advances.

The lectures in this volume are mainly centered around computational approaches. Due to the steadily increasing power of modern capability computers combined with significant algorithmic improvements over the past few years, computational physics/chemistry/biology is by now the third column of basic research tools in fundamental natural science besides the traditional analytical theory and experiments. This is nicely illustrated by the "Landau triangle" in Fig. 13.1 of Chap. 13.

Among the most prominent joint key features of the three fields considered in this volume are rugged free-energy landscapes which generate metastability and are often responsible for very slow dynamics. In computer simulation studies, this is reflected by a slow time evolution of the systems which tend to get trapped in one of these multiple minima. It is a great challenge to develop new simulation algorithms that alleviate the resulting slowing down problem. Usually a quite specific knowledge of the considered system is necessary to develop ideas for improvements. Once discovered, however, it is often possible to transfer new methods also to the related fields. In the last couple of years, precisely this mechanism has been at work concerning the rugged free-energy 
landscape problem, where improvements have been discovered (and often also rediscovered) in computational physics, computational chemistry and more recently also computational biology.

To follow up these developments and take advantage of them, a fairly deep understanding of at least the basic notions and elementary properties of the specific systems is necessary. For example, the concept of "overlap parameters" is rooted in the replica theory of spin glasses, originally developed for infinite-range mean-field models of the Sherrington-Kirkpatrick type. Later the computational definition of "real replicas" and their "overlap parameters" has been carried over to short-range spin glasses of the Edwards-Anderson type. More recently, "overlap parameters" are considered in a by now quite routine fashion also in protein-folding studies to measure the distance to some given reference conformation.

Besides similarities and analogies there are, of course, also many features that distinguish the three systems. For example, the overall shapes of the free-energy landscape for the protein-folding problem and for spin glasses are quite different, see Fig. 1.1. While for proteins a funnel-like landscape with a minimum hosting the native conformation is expected, spin glasses exhibit pronounced metastability. The main difference between glasses and spin

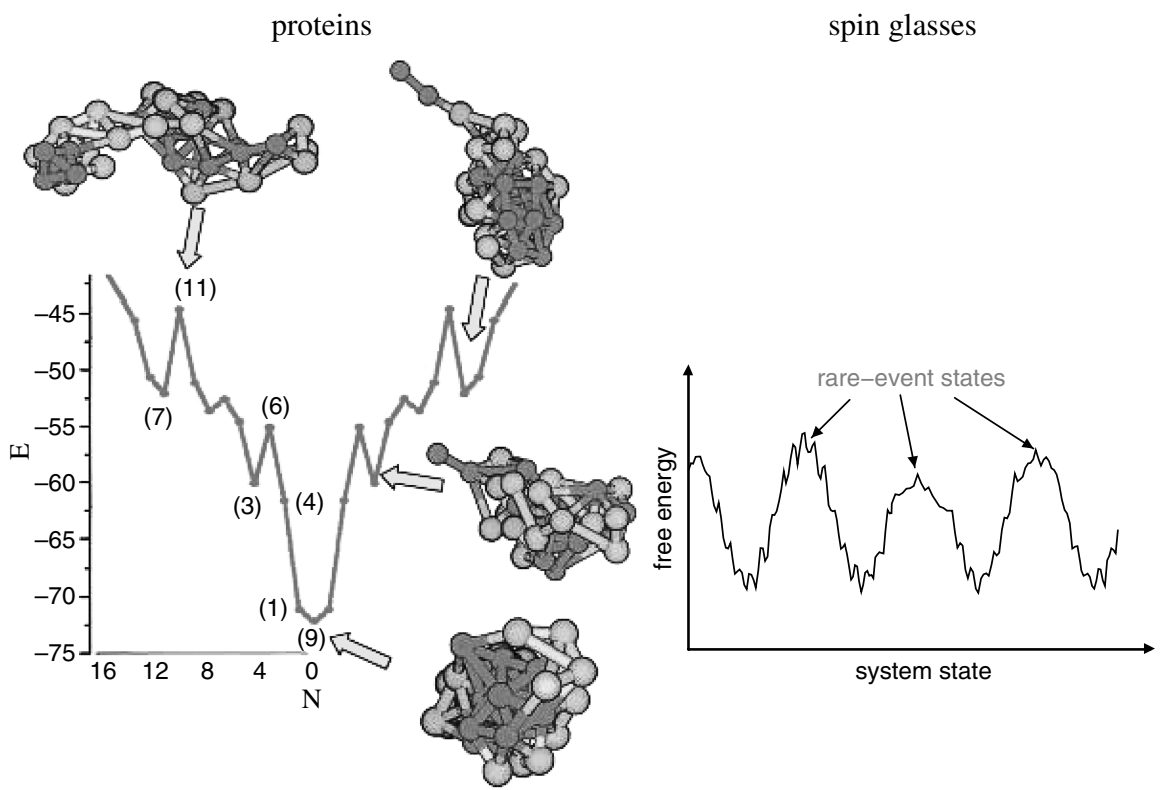

Fig. 1.1. Typical shapes of rugged free-energy landscapes in the protein-folding problem and for spin glasses. While the native protein conformation resides in the minimum of a funnel-like landscape, for spin glasses many degenerate minima are expected (the protein sketch is taken with permission from G. Srinivas, B. Bagchi: J. Chem. Phys. 116, 8579 (2002)) 
glasses is that the glassiness of the former is caused intrinsically by structural disorder while the latter are governed by quenched, random couplings which introduce frustration and disorder. The purpose of this volume is, therefore, to stress the conceptual similarities and differences of these physically quite diverse phenomena and to prepare the reader for adopting useful methods from a neighboring field by providing introductory lecture notes explaining the basic principles of each of the three disciplines.

Part I on spin glasses $[1,2]$ starts in Chap. 2 with a lecture by A. Billoire, who first gives an introduction into the phenomenology of spin glasses and then explains the differences between mean-field Sherrington-Kirkpatrick and short-range Edwards-Anderson Ising spin-glass models. Next he sketches the celebrated replica field theory and discusses the controversy between the resulting replica and alternative droplet picture, which is still not yet resolved. In the main part of his lecture notes, he focuses on Monte Carlo computer simulations of the Sherrington-Kirkpatrick model, for which by now many exact (and partly even rigorous) results are available. In the data analysis, he pays particular attention to finite-size effects, which are of tremendous importance for any serious computer simulation study.

Chapter 3 is devoted in its main part to a special mean-field spin glass model, the 10-state Potts glass, where both a static and a dynamic transition occur [1]. K. Binder, C. Brangian and W. Kob discuss in their lecture notes to what extent the transitions of this model resemble the behavior of a glassforming fluid, that is the transition to a structural glass [3, 4]. Next they turn their attention to Monte Carlo computer simulations of the 10-state Potts glass with nearest-neighbor interactions and provide numerical evidence that no trace of the static and dynamic glass transition observed in the mean-field limit is left in the short-range case.

In Chap. 4, A. K. Hartmann gives an in-depth introduction to exact methods for finding the ground state and the first excited states of two-dimensional Ising spin glasses. Here graph-theoretical concepts such as minimum-weight perfect-matching algorithms are explained and applied to analyses of domain walls, droplets and free-energy barriers [5]. One important conclusion is that assumptions underlying the droplet picture can be verified numerically. In the last section of this chapter, the graph-theoretical approach is extended to dynamical properties such as the scaling of the energy barriers with the size of the system.

Dynamical scaling properties and ageing in disordered systems far from equilibrium are the main subject of Chap. 5, where M. Henkel and M. Pleimling give a comprehensive overview of recent achievements [6]. Starting from simple non-glassy ferromagnets quenched to below the Curie temperature, they discuss ferromagnetically disordered magnets and spin glasses at criticality. In the latter cases, the dependence of the scaling properties on the distribution of the random, quenched couplings is carefully examined. As their main theme, particular attention is devoted to the question to what extent a recently proposed generalization of dynamical scaling to a local scale invariance (LSI) can 
be confirmed by numerically obtained data. Two extensive tables covering a wide range of models and one collecting experimental results provide invaluable information on the state-of-the-art concerning coarsening kinetics and other dynamical properties.

In Part II on structural glasses [3, 4], J. Horbach begins in Chap. 6 with a summary of recent molecular dynamics (MD) computer simulations of various sodium silicate melts. Here the concept of percolating sodium-rich channels is introduced, which form a channel network that can be detected numerically and also experimentally by a prepeak in the static structure factor. On the theoretical side, the framework of mode-coupling theory (MCT) is discussed and applied to the calculation of coherent and incoherent scattering functions, which are explained in this article. The results are found in qualitative agreement with direct "measurements" within the molecular dynamics simulations.

Chapter 7 is devoted to a somewhat exotic and, at least in the condensedmatter community, less well-known spin model, the so-called gonihedric Ising model in three dimensions. In fact, historically this model had its genesis as a possible discretization of string theory in high-energy physics. Starting from the Nambu-Goto formulation over its generalization by an additional extrinsic curvature term or alternatively through the Steiner model, one eventually ends up with a discretized lattice model of the Ising type with next-to-nearest neighbor and plaquette interactions. By tuning the respective coupling constants in a way suggested by the string theory perspective, one obtains a lattice model without quenched coupling constants that nevertheless does exhibit genuine glassy behavior. In this sense, the "self-induced" glassiness resembles rather a structural than a spin glass $[1,2,3,4]$.

Part III of this volume deals with biological macromolecules and in particular with the computationally very demanding protein-folding problem $[7,8]$. Four lecture notes take the reader through the whole hierarchy of descriptions on different length scales of resolution, from coarse-grained lattice and offlattice models over simplified all-atom formulations to detailed atomistic models incorporating empirical force fields such as AMBER, CHARMM, ECEPP, GROMOS, etc.

In Chap. 8, M. Bachmann and I start at the coarsest level and explain in detail mesoscopic model formulations of the hydrophobic-polar type, where the 20 naturally occurring amino acids are classified into only two classes, "hydrophobic" (trying to avoid the aqueous solvent) and "polar" or "hydrophilic" (being favorably attracted by the polar solvent). When the heteropolymer chains are modeled as self-avoiding walks on a lattice, this is the so-called HP model, and for chains in the continuum with bending energy one speaks of the AB model. Using these coarse-grained formulations, computer simulation studies of protein folding and aggregation as well as of the adsorption propensity of peptides to solid substrates are discussed. Compared with detailed all-atom formulations, the simplicity of the coarse-grained models allows for a more universal description and classification of structure formation 
processes resulting in various conformational pseudophases. On the methodological side, various variants of multicanonical chain-growth algorithms with population control are introduced for the lattice models and general ensemble techniques (multicanonical, parallel tempering, etc.) are employed in the continuum formulations.

Exact energy landscapes of proteins within a suitably defined coarsegrained model is the main topic of the lecture notes by F. Dressel and S. Kobe in Chap. 9. They point out that not only calculating the ground state of a protein model but also its excited states with the correct energies is a very promising but still challenging task. To tackle this problem, the use of an exact branch-and-bound algorithm is proposed that can map the whole landscape below a certain energy threshold. This allows the characterization of all states and transitions between them. As an application, results for both globular and membrane proteins are presented and compared with atomic force microscopy experiments on membrane proteins.

In Chap. 10, A. Irbäck presents his all-atom model with simplified and computationally convenient interaction potentials and implicit solvent. In contrast to detailed all-atom models, this potential is calibrated against data pertaining to folding properties of whole chains, rather than of small groups of atoms. The current version of the PROFASI simulation package is able to fold several sequences with about 20 amino acids, including both peptides with $\alpha$-helical and $\beta$-sheet structures. Furthermore, it is demonstrated that the simulation package can reproduce the mechanical unfolding behavior of ubiquitin, a 76 amino acid protein, and finally also a successful study of the aggregation properties of the 7-amino acid fragment $A \beta_{16-22}$ of the amyloid- $\beta$ peptide associated with Alzheimer's disease is presented.

All-atom simulations of proteins using the ECEPP energy function as implemented in the SMMP simulation package are reviewed by U. H. E. Hansmann in Chap. 11. Here, also the energy landscape paving optimization algorithm "ELP" is explained, which is closely related to generalized ensemble simulation techniques, in particular in the formulation discussed by D. P. Landau in his lecture notes in Chap. 13. After reviewing applications of parallel tempering, multicanonical sampling and simulated tempering to biological macromolecules [9], as an example, the peptide with one-letter code EKAYLRT is discussed, which appears in naturally occurring proteins with significant frequency at positions of both $\alpha$-helices and $\beta$-sheets, as well as the 36-residue villin headpiece subdomain HP-36, which is one of the smallest peptides that can fold autonomously.

Part IV on algorithmic developments begins in Chap. 12 with an introduction to Markov chain Monte Carlo methods by B. A. Berg. Starting out with the general idea of importance sampling and its realization by Markov chains $[10,11]$, local update rules such as the Metropolis and heat-bath algorithm are explained next. The basic methods are illustrated with relatively simple example simulations of Ising, Potts and $O(3)$ spin models on regular lattices. Special emphasis is put on a thorough analysis of statistical errors 
of Monte Carlo data, including autocorrelation times as well as binning and Jackknife techniques, which is a "must" for any serious computer simulation study. Next generalized ensembles for Monte Carlo simulations are presented and a detailed exposition of the various tricks along the way is given. Here as application also examples from protein folding are discussed. The article closes with a section on biased Markov chain Monte Carlo methods such as the "Rugged Metropolis" algorithm, which may find useful applications to biophysics in the future.

In Chap. 13, D. P. Landau presents a more recently developed generalizedensemble algorithm, where a random walk in energy space is performed and where the resulting density of states is modified continuously to produce a "flat" histogram. The properties of this method are first illustrated for twodimensional Ising and Potts models, where exact or highly accurate numerical results are available for comparison. Also more elaborate applications of this so-called Wang-Landau sampling to spin glasses and biological macromolecules are sketched, and many other applications to classical and quantum statistics problems are briefly outlined.

The lecture notes by Y. Sugita, A. Mitsutake and Y. Okamoto in Chap. 14 give finally a comprehensive overview of generalized-ensemble algorithms with special emphasis on applications to biological macromolecules [9]. Here most implementations are discussed for both Monte Carlo and molecular dynamics simulations. The authors discuss the multicanonical algorithm, simulated tempering and the replica-exchange or parallel tempering method as well as several powerful combinations thereof. In three comparative case studies, they investigate the crucial question which of the various methods is the most recommendable for protein-folding studies, both with implicit and explicit solvent molecules.

\section{Acknowledgements}

I wish to thank all authors for devoting their time to these lecture notes and Dr. Christian Caron from Springer-Verlag for very patiently accompanying and supporting this project.

\section{References}

1. K. Binder, A. P. Young: Spin glasses: experimental facts, theoretical concepts, and open questions, Rev. Mod. Phys. 58, 801 (1986)

2. A. P. Young (ed.): Spin Glasses and Random Fields (World Scientific, Singapore, 1998)

3. K. Binder, W. Kob: Glassy Materials and Disordered Solids - An Introduction to Their Statistical Mechanics (World Scientific, London, 2005)

4. E.-W. Donth: The Glass Transition. Relaxation Dynamics in Liquids and Disordered Materials (Springer, Berlin, 2001) 
5. A. K. Hartmann, M. Weigt: Phase Transitions in Combinatorial Optimization Problems (Wiley-VCH, Weinheim, 2005)

6. M. Henkel, M. Pleimling, R. Sanctuary (eds.): Ageing and the Glass Transition, Springer Lecture Notes in Physics 716 (Springer, Berlin, Heidelberg, 2007)

7. T. E. Creighton: Proteins: Structure and Molecular Properties (Freeman, New York, 1993) 2nd ed.

8. C. Branden, J. Tooze: Introduction to Protein Structure (Garland, New York, 1999) 2nd ed.

9. U. H. E. Hansmann, Y. Okamoto: The generalized ensemble approach to protein folding simulations. In D. Stauffer (ed.): Annual Reviews in Computational Physics VI (World Scientific, Singapore, 1999), p. 129

10. B. A. Berg: Markov Chain Monte Carlo Simulations and Their Statistical Analysis (World Scientific, Singapore, 2004)

11. D. P. Landau, K. Binder: A Guide to Monte Carlo Methods in Statistical Physics (Cambridge University Press, Cambridge, MA, 2005) 2nd ed. 